\title{
Genetic factors in threatened species recovery plans on three continents
}

Jennifer C Pierson ${ }^{1,2 *}$, David J Coates ${ }^{3}$, J Gerard B Oostermeijer ${ }^{4}$, Steven R Beissinger ${ }^{5}$, Jason G Bragg ${ }^{6}$, Paul Sunnucks ${ }^{7}$, Nathan H Schumaker ${ }^{8}$, and Andrew G Young ${ }^{1}$

Around the world, recovery planning for threatened species is being applied in an attempt to stem the current extinction crisis. Genetic factors linked to small population processes (eg inbreeding, loss of genetic diversity) play a key role in species viability. We examined how often genetic factors are considered in threatened species recovery planning. We selected recent species recovery plans from Europe $(n=110)$, North America (the US only; $n=100)$, and Australia $(n=108)$, and reviewed three broad categories of genetic data they address: population-genetic, fitness-related, and life-history data. We found that the host country, taxonomic group to which the species belonged, and several proposed management actions were important predictors of the inclusion of genetic factors. Notably, species recovery plans from the US were more likely to include genetic issues, probably due to legislative requirements. We recommend an international standard, similar to an IUCN Red List framework, that requires explicit consideration of genetic aspects of long-term viability.

Front Ecol Environ 2016; 14(8): 433-440, doi:10.1002/fee.1323

$\mathrm{T}$ he goal of species recovery planning is typically to restore and maintain viable populations (Doak et al. 2015). Many threatened species occur in small, isolated populations, where genetic and demographic processes substantially influence population and species viability (Pierson et al. 2015). There is widespread evidence that (1) genetic factors such as inbreeding depression (Frankham 2010) and loss of genetic diversity (Spielman et al. 2004) influence extinction risk and (2) genetic rescue of inbred populations through gene flow restores their viability

\section{In a nutshell:}

- Despite their importance in helping to ensure the persistence of wild populations, genetic factors are frequently absent from conservation and management initiatives

- We reviewed over 300 threatened species recovery plans from seven countries; US plans and those associated with animals (rather than plants) contained the most genetic information

- An international standard needs to be developed, explicitly stating how these plans should include genetic factors in recovery efforts

- Greater awareness and acceptance of the value of integrating genetic considerations in species recovery planning is also necessary

\footnotetext{
${ }^{1}$ Commonwealth Scientific and Industrial Research Organisation (CSIRO) Plant Industry, Canberra, Australia; ${ }^{2}$ The Fenner School of Environment and Society, The Australian National University, Canberra, Australia*(jennifer.pierson@anu.edu.au); ${ }^{3}$ Science and Conservation Division, Department of Parks and Wildlife, Perth, Australia; ${ }^{4}$ Institute for Biodiversity and Ecosystem Dynamics, University of Amsterdam, Amsterdam, The Netherlands; continued on $p 440$
}

(Frankham 2015). However, in practice, many conservation and management initiatives have yet to include genetics findings (Waples et al. 2008; Laikre et al. 2010).

Species recovery plans are an excellent resource to gauge the inclusion of conservation research into management, specifically to assess whether genetic factors are being considered when managing small populations. Although 22\% of 90 US endangered and threatened animal species recovery plans (1977-1998) identified genetic threats to population persistence, these threats were rarely incorporated into specific recovery or management tasks (Moyle et al. 2003). However, in recent decades, theoretical and technological advances have greatly improved and broadened the application of genetics to conservation issues (Allendorf et al. 2010; Funk et al. 2012; Harrisson et al. 2014). Despite these scientific advances, the explicit inclusion of genetic information in management actions often continues to lag behind other information used in species management (Laikre et al. 2009). For example, Waples et al. (2008) looked at reasons why uptake of genetic data has been slow in fisheries management and identified important communication and institutional issues that need to be overcome. Indeed, many of the instances where genetic data have been integrated into conservation have not been published in the peer-reviewed literature, making it difficult to quantify its successful implementation in population management (Garner et al. 2016).

Here, we assess the inclusion of genetic factors in threatened species recovery plans, which provide an objective, published record of intended management actions and thus allow quantification of the uptake of genetic data in management. Recovery plans often deal with small populations for which genetic factors 
(eg inbreeding depression, small effective population size $\left.\left[N_{e}\right]\right)$ are likely to be a concern and where genetics has the potential to help surmount practical management challenges such as monitoring recruitment, dispersal, and population trends (Schwartz et al. 2007). Because recovery plans are usually created in response to specific legislation (Gibbs and Currie 2012) and policy, we assessed plans from three distinct regions - the US, Europe, and Australia - that we hypothesized would address genetic criteria in different ways, given the regional differences in legislation and policy. All three regions are economically developed and are sufficiently wealthy to have substantial conservation planning in place, yet may be dealing with different threats to at-risk species. Over the centuries, Europe has been characterized by high human population density along with fragmented habitats and reduced wildlife population sizes (Dullinger et al. 2013). The US and Australia have experienced more recent conservation challenges, with many species declining and becoming imperiled after European colonization, beginning in the 15 th and 17 th centuries. In the US, the greatest threats are habitat loss and fragmentation (Wilcove et al. 1998). Australia has lower human population densities than Europe and the US but has seen negative environmental impacts from numerous introductions of non-native species in addition to habitat loss and fragmentation due to rapid, massive land-clearing (Underwood et al. 2009). Given the potential differences between species' lifehistory strategies and perceived charisma of some species (ie plants versus animals) within these three parts of the world, we also hypothesize that the inclusion of genetic risks and/or genetic data in plant and animal species recovery plans may vary between these areas.

We examined three types of genetic factors that could be included in recovery plans: (1) population-genetic processes, (2) fitness-related parameters, and (3) lifehistory attributes. Population-genetic processes affect patterns of genetic variation that can provide ecological information on connectivity and gene flow, patterns of mating within populations, and levels of population contraction and expansion. Fitness-related parameters, such as inbreeding and hybridization, can be directly related to population viability (Spielman et al. 2004; Leimu et al. 2006). These parameters are not fixed but result from current and historical population processes and can be directly targeted for management; they can also be a primary contributor to species becoming caught in an "extinction vortex" (Blomqvist et al. 2010) - once interacting factors produce a vortex of decline, abatement of threats that caused the initial decline may not be enough to ensure recovery (Hutchings et al. 2012). Life-history attributes, such as mating systems, are relatively fixed traits that can also influence fitness processes. For example, many plants are self-incompatible and their mate availability depends on diversity for specific genes (eg the S-locus; Pierson et al. 2013). Management of this diversity can be fundamental to population recovery.
For each of the reviewed plans, we evaluated how genetic issues are incorporated into preparations for threatened species recovery, testing what background information and proposed actions best predicted the presence of genetic factors or inclusion of genetic data. We determined whether collection of genetic data was included or recommended, and, if so, which of the three types of genetic factors (listed above) were taken into account. We also investigated recovery actions related to genetic aspects of population or species viability. Finally, we examined whether there were differences among regions and (within Europe) countries, as well as between recovery plans focused on animal and plant taxa.

\section{Methods}

\section{Genetics in recovery plans}

We reviewed 318 threatened species recovery plans (WebTable 1), which included 100 from the US, 108 from Australia, and 110 from five European countries (France, the Netherlands, the UK, Germany, and Luxembourg). We converted most results into percentages to facilitate comparisons. Because there may be differences between recovery plans for plants and animals, we included the $\sim 50$ most recent single-species plans from each taxon in each region. The US plans were identified and sampled from the US Fish and Wildlife Service's online Recovery Plan database (http:// bit.ly/29PhgAR). In Europe, recovery plans for threatened species are government-mandated at the national level in France (http://bit.ly/2a6pW8E), Luxembourg (http://bit.ly/2ao03QW), and the UK (jncc.defra.gov. uk/page-5161). In Germany, they are coordinated at the county level (eg Brandenburg [http://bit.ly/29Q $9 \mathrm{YxH}$ ] and Bayern [http://bit.ly/2ab1iXw]). In the Netherlands, species conservation plans are generally initiated by non-governmental organizations (NGOs) for specific taxonomic groups, and are funded by the national or provincial government. In cases where $>50$ recovery plans were listed for a taxon/region combination, we drew a random sample from the list. Australian recovery plans were sampled from the Australian Government Department of the Environment's species profile and threats database (http://bit.ly/29Vxv35).

We developed a standard questionnaire modeled after a previous review of endangered species recovery plans in the US (WebTable 2; Hoekstra et al. 2002) and applied it to the electronic versions of recovery plans collected via online searches. For each plan, we recorded background data, including region and country of origin, year finalized, plant versus animal and taxonomic category (fish, reptiles and amphibians, birds, mammals, insects, herbaceous annuals, herbaceous perennials, or woody plants). Because - in all three regions - recovery plans are generally based on non-binding recommendations, this may influence both the content and rigor of proposed actions. 
We examined whether genetic issues were incorporated in any form in the recovery plan and if genetic data were included (I) and/or if tasks ( $\mathrm{T}$ ) were assigned to collect genetic data (IT $=$ both data included and tasks assigned). We then asked whether specific types of genetic factors were considered in recovery plans: population-genetic parameters (within-population genetic variation, genetic structure, gene flow among populations, and $N_{e}$ ), fitnessrelated parameters (estimates of inbreeding using molecular markers, inbreeding depression, hybridization and/or introgression, and outbreeding depression), and lifehistory related parameters (mating system, chromosome variation [plants only], and clonal propagation [plants only]). Finally, we looked at whether management actions were assigned that benefit directly from genetic information: (1) reintroduction or translocation, (2) ex situ preservation of genetic resources, which could include cryostoring gametes or embryos as well as seed banking, and (3) captive breeding or maintaining seed orchards.

We used chi-square contingency tests to determine if there were differences among the three regions or between plant and animal plans in terms of whether genetic factors were included and whether genetic data were collected or whether such data collection was planned. We used a generalized linear model with a binomial distribution and logit link to assess factors predicting whether genetic aspects were considered and genetic data were collected/planned, both of which were used as response variables. Explanatory variables included background information and recovery actions that benefit from the inclusion of genetic information (WebTable 3, $\mathrm{a}$ and $\mathrm{b}$ ). Nested variables (eg region and country) were not included in the same models.

We used Akaike's Information Criterion (AIC) to evaluate support for our suite of models. Because there were nested variables (region/country and plant versus animal/taxonomic category), we began running each model with a single explanatory variable, and included in the global model only the variable from the nested pair that had more individual support (lower AIC). We also omitted single variables from the global model that did not improve model fit as compared with the null model (intercept only). Once the global model was defined, the best model was chosen using a backward-selection approach (stepAIC function in the MASS library in R). We assessed the goodness-of-fit of the global model using Nagelkerke's $R^{2}$, a pseudo $R^{2}$ used to assess fit of logistic models by comparing the ratios of likelihoods of the full model and the intercept model (fmsb package in R). All analyses were performed in R (R Core Team 2013).

\section{Results}

\section{Are genetic factors seen as a risk?}

Overall, approximately one-half (49.6\%) of the sampled plans regarded genetic factors a risk in population recovery. There was a strong relationship between the plan's region of origin and the consideration of genetics as a risk factor $\left(\chi^{2}=19.7, P<<0.001\right)$, with $63 \%$ of US plans, $55 \%$ of Australian plans, and 33\% of European plans regarding genetic factors a risk (Figure 1). Recovery plans focused on animals viewed genetic factors a risk factor $(57 \%)$ significantly more often than did plans focused on plants $(43 \%)\left(\chi^{2}=3.92, P=0.048\right)$, which appears influenced mainly by Australian plans.

When we tested which factors predicted whether genetic issues were included in a recovery plan, the bestfitting model (Nagelkerke's $R^{2}=0.39 ; 24.8 \%$ deviance explained) included the country of origin, taxonomic category, and two management actions: (1) reintroduction or translocation and (2) preservation of genetic resources ex situ (WebTable 3a). A model within two AIC points of the best-fitting model included plans for captive breeding or a seed orchard, but this variable was not significant and had a small effect size (coefficient $=$ $0.32 ; P=0.33$ ) so we did not consider it further. Countryof-origin effects appear to be influenced not only by France, where genetic factors were considered more often than in other European countries and Australia, but also by the UK and Luxembourg, where genetic issues were included less often than in Australia and the US (Figure 2; WebTable 4a). Effects due to taxonomic differences were

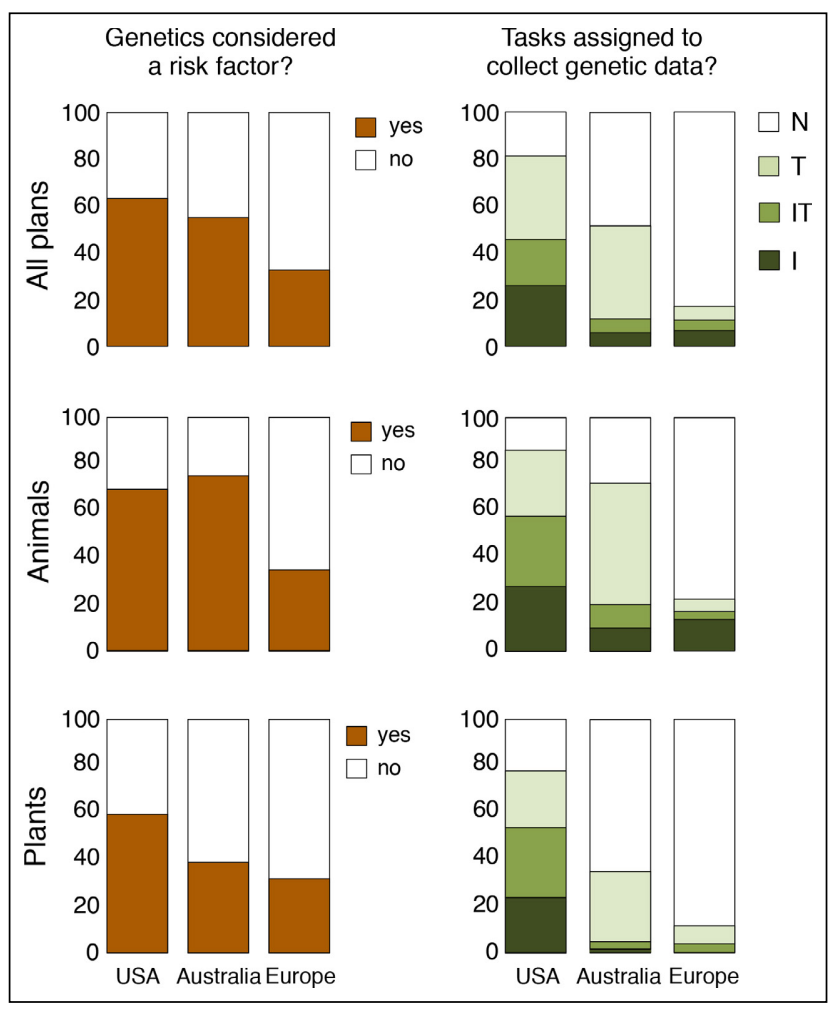

Figure 1. Summary of threatened species recovery plans that consider genetic factors as a risk to population viability and then either present data $(I=$ included $)$ or assign tasks $(T=$ tasks assigned) to collect genetic data. IT = plans both include and assign tasks to collect data; $N=$ none specified. 


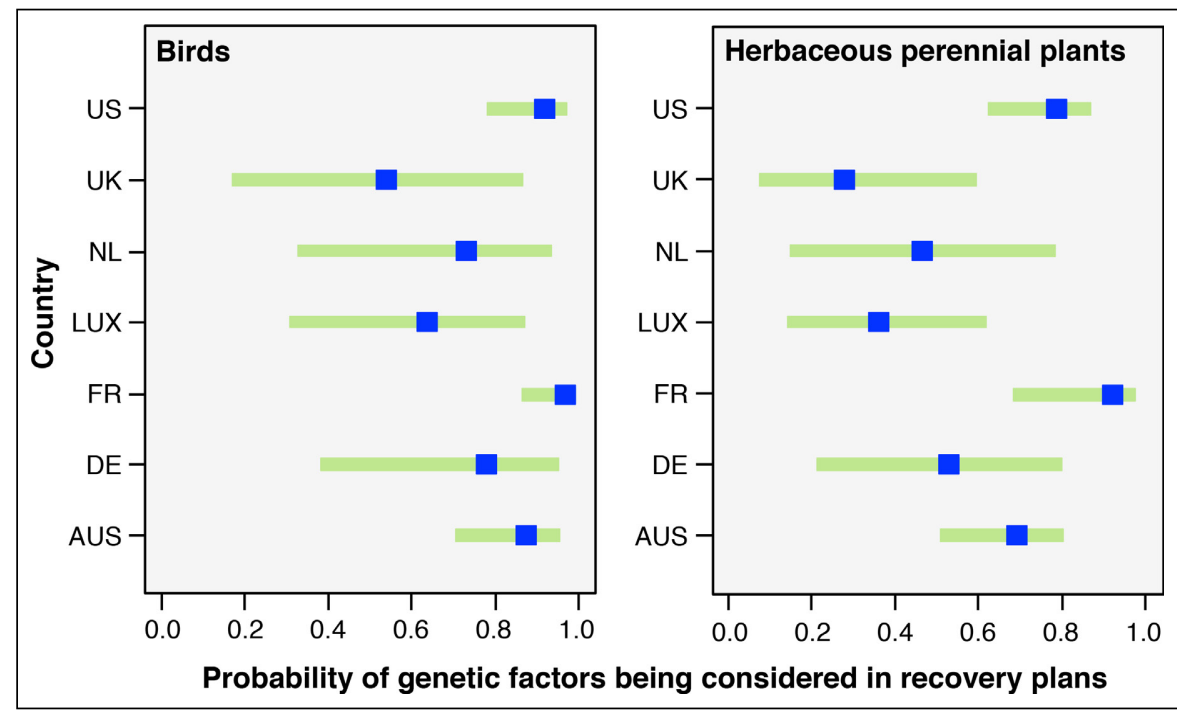

Figure 2. The predicted values (blue squares) and 95\% confidence intervals (green lines) based on the best-fitting model, which included "country", "taxa", "reintroduction or translocation", and "preservation of genetic resources ex situ", of the probability of genetic factors being considered. We provide an example of animals (birds) and plants (herbaceous perennials) and in each case hold both reintroduction and genetic resources ex situ as "yes".

influenced mostly by amphibians and reptiles, for which genetic factors were considered more often, and by all plant categories, which incorporated genetic factors less often (Figure 2; WebTable 4a).

\section{Is collection of genetic data included or recommended?}

Overall, nearly one-half (49.2\%) of plans included genetic data and/or assigned tasks to collect them. There was a strong relationship between the region and collection of genetic data $\left(\chi^{2}=84.7, P<0.001\right)$, with $82 \%$ of US plans, $52 \%$ of Australian plans, and 17\% of European plans including genetic data and/or assigning tasks to collect them. In the US, $46 \%$ of plans already included some type of genetic data, but few Australian (12\%) and European (11\%) plans did (Figure 1). Animal recovery plans also included or planned to collect genetic data significantly more often than plant recovery plans $\left(\chi^{2}=6.5, P=0.011\right)$. The general significant result is mainly attributable to Australia, where a larger number of animal recovery plans took into account and/or included genetics (Figure 1).

The best-fitting model (Nagelkerke's $R^{2}=0.66 ; 49.8 \%$ deviance explained) for whether recovery plans recommended genetic data be collected included country of origin, taxonomic category, genetic factors considered in the plan, and reintroduction or translocation plans (WebTable 3b). However, models that included year finalized and captive breeding or seed orchard management actions were within approximately two AIC points of the best model. Both variables had small effect sizes (coefficient $=0.07$ and 0.01 , respectively) and were not significant $(P=0.21$ and 0.98 , respectively) so were not considered further. Taking into account genetic factors in recovery plans was strongly positively associated with the inclusion or planned use of genetic data. Country-of-origin patterns included Germany, which did not have any plans that included or assigned collection of genetic data. Luxembourg, the Netherlands, and the UK also had few plans that collected genetic data, whereas the US included or assigned collection of genetic data more often (all compared to Australia as a reference; Figure 3). France was the only European country that included or assigned collection of genetic data at a similar, relatively high, rate as Australia (Figure 3). Taxonomic category was greatly influenced by mammal recovery plans that included or planned genetic data collection more often (compared to birds as a reference), and insects, woody plants, and herbaceous annuals less often. Finally, management actions involving reintroduction or translocation were positively but weakly associated with the inclusion or assigned collection of genetic data.

\section{What type of genetic factors are considered?}

Population-genetic factors were included most often, particularly genetic variation within populations and genetic structure (Figure 4). The inclusion of population genetic variation was common for animal plans in the US (70\%) and Australia (60\%) but much less so in Europe $(12 \%)$. Data on gene flow among populations and $N_{e}$ were included or planned less often $(18 \%$ and $11 \%$ of all plans, respectively).

Life-history factors were the next most-considered category (WebFigure 1). Of these, breeding behavior or mating system were most commonly included (24\%), most often in plant plans and animal plans from the US (67\% and $34 \%$, respectively). In Australia, only $2 \%$ of plant recovery plans considered mating systems. Clonal propagation was covered in only $5 \%$, and chromosome variation in only $0.3 \%$ of all plant plans, regardless of region.

Fitness-related factors were seldom considered (Figure 5). Only 7\% of plans included or included use of molecular markers to estimate inbreeding, and only $6 \%$ of plans included or planned to collect inbreeding depression data. Hybridization/introgression and outbreeding depression were included even less often (in 5\% and 1\% of all plans, respectively). 


\section{Are genetic aspects of population viability recommended?}

Among the three recovery actions that were evaluated, translocations and reintroductions were considered most often $50 \%$ of reviewed plans), followed by the ex situ preservation of genetic resources (39\%), and captive breeding or seed orchards (32\%) (Figure 6).

Translocations and/or reintroductions were recommended for a majority of plans in the US and nearly one-half of Australian plans (49\%), with plant plans contributing most to this pattern (Figure 6). A high proportion of Australian plans $-84 \%$ of plant plans and $36 \%$ of animal plans - recommended ex situ preservation of genetic resources (Figure 6). US plans recommended the ex situ preservation of genetic resources for $60 \%$ of plant species but rarely recommended this for animal species (4\%) (Figure 6). Overall, captive breeding or seed orchards were recommended in $<40 \%$ of the plans in each region (Figure 6). In the US and Australia, captive breeding was recommended in $46 \%$ and $49 \%$ of animal plans, respectively, and seed orchards were recommended in $43 \%$ and $45 \%$ of plant plans, respectively. Fewer European plans recommended captive breeding (17\% of animal plans) or seed orchards (34\% of plant plans).

\section{Discussion}

Of the threatened species recovery plans that we reviewed, genetic factors were sometimes considered but genetic data were seldom included. Some plans involved collection of genetic data, but this was highly dependent on the region. The large proportion of US plans that included genetics is encouraging, given that previous reviews found the inclusion of genetic considerations or data in US recovery planning to be "limited and ill-defined" (Moyle et al. 2003) and highly variable (Fallon 2007). The low proportion in Europe is in line with the observed lack of input by conservation scientists, in particular geneticists, in the interpretation of the European Union (EU) concept of Favourable Conservation Status (FCS) of endangered species (Laikre et al. 2009).

Most recovery plans covered population-genetic factors that did not necessarily inform fitness directly. $N_{e}$, a fundamental parameter for assessing population viability, was considered in only $\sim 10 \%$ of all plans. Given the central importance of this parameter to understanding the conservation of populations in both the short and long term (Jamieson and Allendorf 2012), it is remarkable how seldom $N_{e}$ is included in threatened species recovery plans.

Generally, fitness-related parameters were overlooked in all plans. Again, this finding is somewhat unexpected, given the long-standing evidence that inbreeding depression negatively affects fitness (Keller and Waller 2002; Biebach and Keller 2009; Frankham 2010, 2015), is a risk to small populations, and tends to be the primary genetic concern in viability analyses (Pierson et al. 2015). Genetic rescue of small populations (Whiteley et al. 2015) is often a goal of recovery actions such as translocations or augmentation (Weeks et al. 2011), yet only 7\% of all plans mentioned inbreeding and even fewer discussed inbreeding depression (Figure 5). Fitness can be reduced when locally adapted populations are mixed (outbreeding depression) or mating occurs between different species (hybridization). Outbreeding depression, while apparently less concerning than inbreeding depression because its occurrence is often predictable and its effects are usually mild and/or transient (Frankham et al. 2011), has been raised in some discussions on management policies regarding seed sourcing for restoration programs and translocations (Breed et al. 2012). Surprisingly, only three plans in total mentioned outbreeding depression and hybridization, even though many plans mentioned translocations and captive breeding/seed orchards.

It is unclear why genetic factors are so seldom thought about in recovery plans but this is likely due to a multitude of reasons, relating to the perceived costs in effort and resources, and the apparent conservation benefits of 


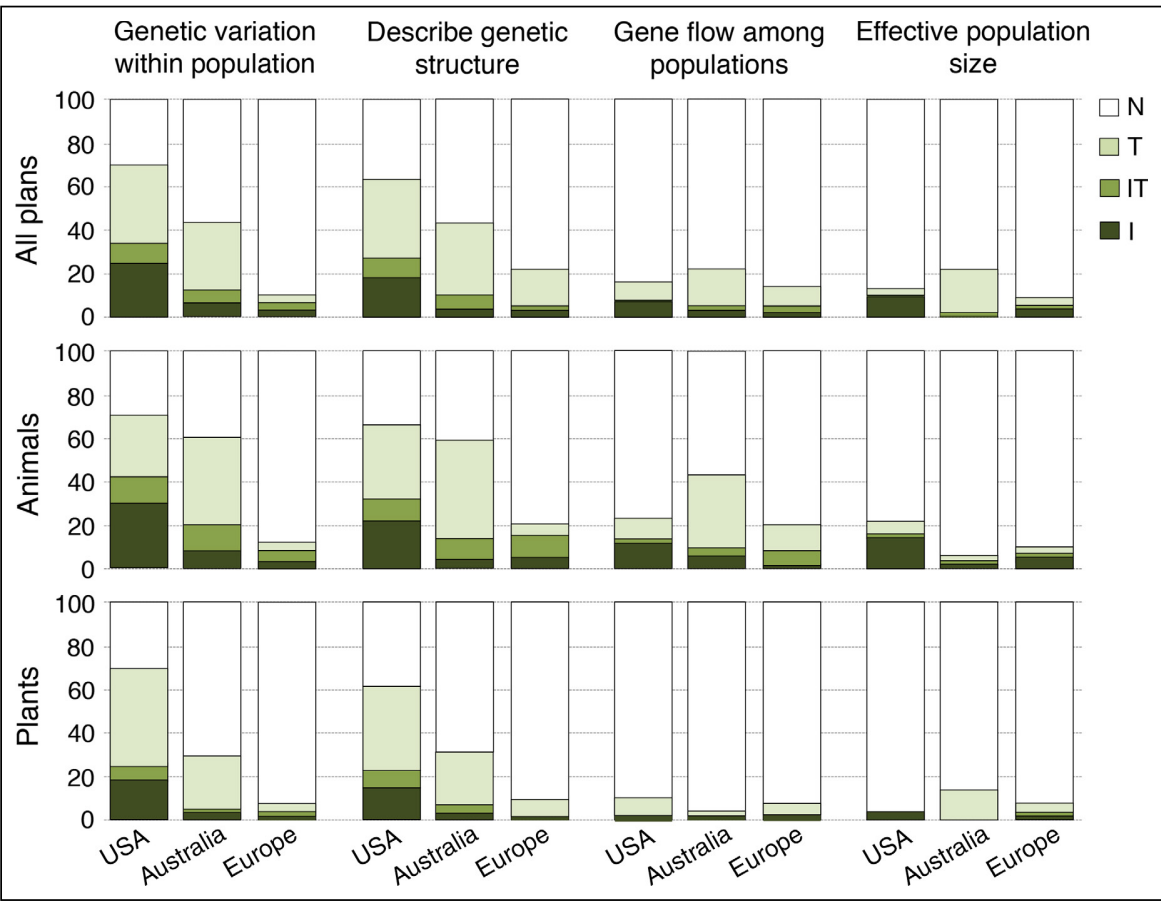

Figure 4. Percentage of recovery plans that include data $(I=$ included $)$ or assign tasks $(T=$ tasks assigned $)$ to collect data on population-genetic factors. IT = plans both include and assign tasks to collect data; $N=$ none specified. have substantial effects on the probability of extinction and persistence, via their impacts on fitness and adaptive potential (Saccheri et al. 1998; Frankham 2010, 2015; Whiteley et al. 2015).

Management actions that should benefit from considering genetic factors were somewhat linked to plans that included genetics. Specifically, reintroduction/translocation and the preservation of genetic resources had a strong relationship with the consideration of genetic factors, which was strongly linked to data collection (WebTable 4, a and b). Nevertheless, the plan's country of origin and the taxonomic group of the organism in question also had a strong influence on the inclusion of genetics in a given recovery plan.

In recovery plans from the US and to some extent from Australia, the focus on inclusion of or collection of genetic data may at least be partially attributed to legislative acquiring genetic information (Vernesi and Bruford 2009). We suspect the benefits of genetic information requirements in these countries. In the US, recovery plans are required to make use of the best possible tend to be underestimated, because the risks posed to threatened species by genetic processes (eg inbreeding depression and loss of genetic diversity) are not sufficiently understood by practitioners. We also acknowledge that genetic factors, although they may not be directly addressed in a recovery plan, could be indirectly assessed through measures of abundance such as population size, and that the likelihood of extinction may be considered in terms of decline in population size and the local extinction of populations through part of a species' range. Poor understanding of genetic risks and any direct reference to these may stem from the inherent complexity of linking genetic data to demographic processes (Waples et al. 2008), and the perception that genetic issues have rarely been shown to be the primary cause of extinction in wild organisms, under natural conditions. However, there is compelling evidence that genetic processes can
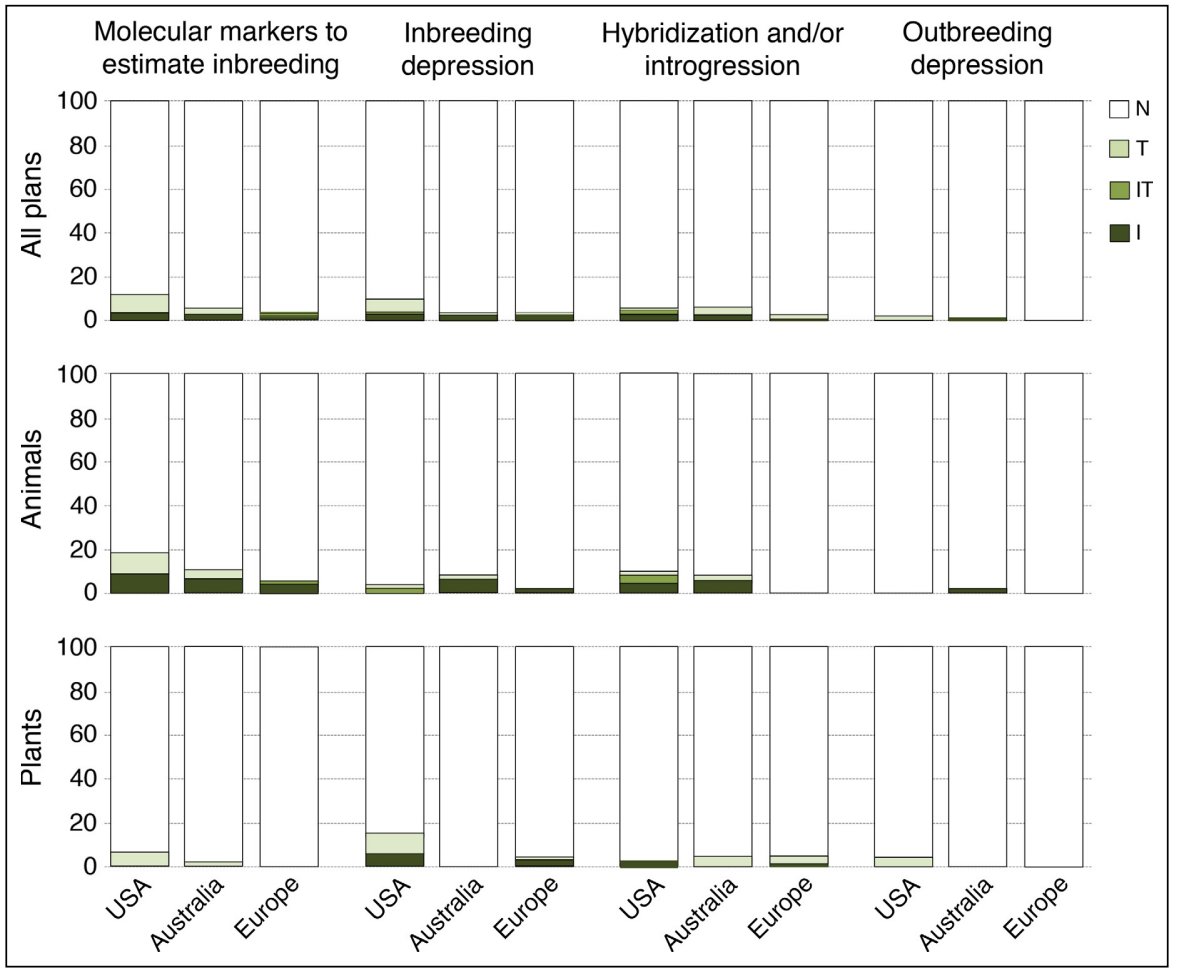

Figure 5. Percentage of recovery plans that include data $(I=$ included $)$ or assign tasks ( $T=$ tasks assigned) to collect data on fitness-related genetic parameters. $I T=$ plans both include and assign tasks to collect data; $N=$ none specified. 
science, and litigation against the federal government plays a role in conservation policy when advocacy groups believe this standard is not being upheld (Noon and McKelvey 1996). In Australia, recovery plans must "provide for the research and management actions necessary to stop the decline of, and support the recovery of, the listed threatened species so that its chances of longterm survival in nature are maximised" (Environment Protection and Biodiversity Conservation Act of 1999, Commonwealth of Australia). In the $E U$, each member state has the responsibility to maintain or ensure FCS (implying long-term metapopulation viability) of all species mentioned in the EU Birds Directive (2009; Directive 2009/ 147/EC, European Parliament) and Habitats Directive (1992; EU Council Directive 92/43/EEC). Other than population size and number, however, there is no formal EU-wide assessment of parameters that would allow (meta)population viability analysis for endangered species, which can be included as a standard for the best possible scientific underpinning of FCS (Mace et al. 2008). Laikre et al. (2009) proposed that genetic parameters form important components of FCS. A subsequent update of the directives recommends consideration of genetic factors more explicitly, but these recommendations still require no commitment (Evans and Arvela 2011).

\section{- Conclusions}

We recommend that the recovery planning process for threatened species include the explicit consideration of genetic factors in all cases. This is not to say that all plans must include genetic data; rather, all plans should openly state how genetic concerns are accounted for (eg inbreeding depression is unlikely to be a concern for a primarily self-pollinating plant). This would certainly improve the current situation, in which genetic factors are often completely ignored - as found in half of all 318 plans we reviewed.

There are several ways to promote the incorporation of genetic factors in recovery planning. First, there needs to be an improved understanding and acceptance of genetic diversity principles in national and international conservation policy and planning. To that end, conservation geneticists should raise awareness with policy makers, government agencies, NGOs, and practitioners that genetic data can provide multiple, cost-effective pieces of information about demographic, genetic and evolutionary processes. Second, an international standard for species recovery plans should be developed that explicitly includes genetic aspects of viability. A good framework is the International Union for Conservation of Nature's (IUCN's) system for classifying threatened species, which uses the same criteria to assess extinction risk of all species, with adjustments made for lifehistory differences such as generation length (Mace et al. 2008). The IUCN's Conservation Genetics Specialist Group could advise on developing such a standard. Finally, several management strategies - including corridors to increase gene flow, population augmentation to reduce genetic erosion, and mixing source populations to maximize genetic diversity in reintroductions - already affect genetic processes. More careful, data-supported consideration of the genetic implications of these actions will help to improve their effectiveness. In conclusion, we advocate for the explicit inclusion of genetic concerns, alongside others, to help ensure successful outcomes for the conservation of imperiled species.

\section{Acknowledgements}

The Australian Centre for Ecological Analysis and Synthesis, CSIRO, and the Western Australian Department of Conservation provided support for this 
research. Assistance with plan reviews was provided by $\mathrm{T}$ Pleines (US plans), S Luijten and Y Hartman (European plans), and S McArthur (Australian plans). We thank A Mortelliti and M Westgate for data analysis and visualization assistance. The US Environmental Protection Agency (EPA) funded NHS and approved this paper for publication; approval does not imply the content reflects the views of EPA.

\section{References}

Allendorf FW, Hohenlohe PA, and Luikart G. 2010. Genomics and the future of conservation genetics. Nat Rev Genet 11: 697-709.

Biebach I and Keller LF. 2009. Inbreeding in reintroduced populations: the effects of early reintroduction history and contemporary processes. Conserv Genet 11: 527-38.

Blomqvist D, Pauliny A, Larsson M, and Flodin LA. 2010. Trapped in the extinction vortex? Strong genetic effects in a declining vertebrate population. BMC Evol Biol 10: 33.

Breed MF, Stead MG, Ottewell KM, et al. 2012. Which provenance and where? Seed sourcing strategies for revegetation in a changing environment. Conserv Genet 14: 1-10.

Doak DF, Himes Boor GK, Bakker VJ, et al. 2015. Recommendations for improving recovery criteria under the US Endangered Species Act. BioScience 65: 189-99.

Dullinger S, Essl F, Rabitsch W, et al. 2013. Europe's other debt crisis caused by the long legacy of future extinctions. P Natl Acad Sci USA 110: 7342-47.

Evans D and Arvela M. 2011. Assessment and reporting under Article 17 of the Habitats Directive. Paris, France: European Topic Centre on Biological Diversity. http://bit.ly/29DRbVM. Viewed 15 Jul 2016.

Fallon SM. 2007. Genetic data and the listing of species under the US Endangered Species Act. Conserv Biol 21: 1186-95.

Frankham R. 2010. Inbreeding in the wild really does matter. Heredity 104: 124.

Frankham R. 2015. Genetic rescue of small inbred populations: meta-analysis reveals large and consistent benefits of gene flow. Mol Ecol 24: 2610-18.

Frankham R, Ballou JD, Eldridge MD, et al. 2011. Predicting the probability of outbreeding depression. Conserv Biol 25: 465-75.

Funk WC, McKay JK, Hohenlohe PA, and Allendorf FW. 2012. Harnessing genomics for delineating conservation units. Trends Ecol Evol 27: 489-96.

Garner BA, Hand BK, Amish SJ, et al. 2016. Genomics in conservation: case studies and bridging the gap between data and application. Trends Ecol Evol 31: 81-83.

Gibbs KE and Currie DJ. 2012. Protecting endangered species: do the main legislative tools work? PLoS ONE 7: e35730.

Harrisson KA, Pavlova A, Telonis-Scott M, and Sunnucks P. 2014. Using genomics to characterize evolutionary potential for conservation of wild populations. Evol Appl 7: 1008-25.

Hoekstra JM, Clark JA, Fagan WF, and Boersma PD. 2002. A comprehensive review of Endangered Species Act Recovery Plans. Ecol Appl 12: 630-40.

Hutchings JA, Butchart SH, Collen B, et al. 2012. Red flags: correlates of impaired species recovery. Trends Ecol Evol 27: 542-46.

Jamieson IG and Allendorf FW. 2012. How does the 50/500 rule apply to MVPs? Trends Ecol Evol 27: 578-84.

Keller LF and Waller DM. 2002. Inbreeding effects in wild populations. Trends Ecol Evol 17: 230-41.

Laikre L, Allendorf FW, Aroner LC, et al. 2010. Neglect of genetic diversity in implementation of the Convention on Biological Diversity. Conserv Biol 24: 86-88.
Laikre L, Nilsson T, Primmer CR, et al. 2009. Importance of genetics in the interpretation of Favourable Conservation Status. Conserv Biol 23: 1378-81.

Leimu R, Mutikainen PIA, Koricheva J, and Fischer M. 2006. How general are positive relationships between plant population size, fitness and genetic variation? J Ecol 94: 942-52.

Mace GM, Collar NJ, Gaston KJ, et al. 2008. Quantification of extinction risk: IUCN's system for classifying threatened species. Conserv Biol 22: 1424-42.

Moyle LC, Stinchcombe JR, Hudgens BR, and Morris WF. 2003. Conservation genetics in the recovery of endangered animal species: a review of US endangered species recovery plans (1977-1998). Animal Biodivers Conserv 26: 85-95.

Noon BR and McKelvey K. 1996. Management of the spotted owl: a case history in conservation biology. Annu Rev Ecol Evol S 27: 135-62.

Pierson JC, Beissinger SR, Bragg JG, et al. 2015. Incorporating evolutionary processes into population viability models. Conserv Biol 29: 755-64.

Pierson JC, Swain SM, and Young AG. 2013. Incest versus abstinence: reproductive trade-offs between mate limitation and progeny fitness in a self-incompatible invasive plant. Ecol Evol 3: 5066-75.

R Core Team. 2013. R: a language and environment for statistical computing. Vienna, Austria: R Foundation for Statistical Computing.

Schwartz MK, Luikart G, and Waples RS. 2007. Genetic monitoring as a promising tool for conservation and management. Trends Ecol Evol 22: 25-33.

Spielman D, Brook BW, and Frankham R. 2004. Most species are not driven to extinction before genetic factors impact them. P Natl Acad Sci USA 101: 15261-64.

Underwood EC, Viers JH, Klausmeyer KR, et al. 2009. Threats and biodiversity in the mediterranean biome. Divers Distrib 15: 188-97.

Vernesi C and Bruford MW. 2009. Recent developments in molecular tools for conservation. In: Bertorelle G, Bruford MW, Hauffe HC, et al. (Eds). Population genetics for animal conservation. Cambridge, UK: Cambridge University Press.

Waples RS, Punt AE, and Cope JM. 2008. Integrating genetic data into management of marine resources: how can we do it better? Fish Fish 9: 423-49.

Weeks AR, Sgro CM, Young AG, et al. 2011. Assessing the benefits and risks of translocations in changing environments: a genetic perspective. Evol Appl 4: 709-25.

Whiteley AR, Fitzpatrick SW, Funk WC, and Tallmon DA. 2015. Genetic rescue to the rescue. Trends Ecol Evol 30: 42-49.

Wilcove DS, Rothstein D, Dubow J, et al. 1998. Quantifying threats to imperiled species in the United States. BioScience 48: 607-15.

\section{Supporting Information}

Additional, web-only material may be found in the online version of this article at http://onlinelibrary. wiley.com/doi/10.1002/fee.1323/suppinfo

\footnotetext{
${ }^{5}$ Department of Environmental Science, Policy and Management, University of California-Berkeley, Berkeley, CA; ${ }^{6}$ Research School of Biology, The Australian National University, Canberra, Australia; ${ }^{7}$ School of Biological Sciences, Monash University, Melbourne, Australia; ${ }^{8}$ Western Ecology Division, Environmental Protection Agency, Corvallis, OR
} 